\title{
The Formation of Young Clusters in Three Ongoing Galaxy Mergers
}

\author{
B. Rothberg ${ }^{1}$, B. Whitmore ${ }^{1}$, F. Schweizer ${ }^{2}$, R. Chandar $^{2}$, \\ R. van der Marel ${ }^{1}$, J. Rossa ${ }^{3}$, W. Blair ${ }^{4}$, S. Mengel $^{5}$, and M. Rieke ${ }^{6}$ \\ ${ }^{1}$ STScI, USA, email: rothberg@stsci.edu, ${ }^{2}$ Carnegie Observatories, USA, ${ }^{3}$ Department of \\ Astronomy, University of Florida, USA, ${ }^{4}$ Department of Physics \& Astronomy, Johns Hopkins \\ University, USA, ${ }^{5}$ ESO, Germany, ${ }^{6}$ Steward Observatory, University of Arizona, USA
}

\begin{abstract}
High-resolution, multi-wavelength (UV through NIR) imaging from the Hubble Space Telescope has been obtained for three mergers in the Toomre Sequence, NGC 520, NGC 2623, \& NGC 3256, to identify and measure the properties of young Globular Cluster (GC) candidates. Unlike previous merger studies, this work focuses on objects that lie in the middle of the Toomre Sequence. This age range represents a critical dynamical transition in the merging sequence between ongoing mergers and advanced remnants with properties more akin to elliptical galaxies. The early results of our investigation include luminosity functions and age-dating of the GC candidates. These results also address the impact of the dynamical state of the merger on the current young cluster population as compared with earlier studies that have dealt with the very oldest and very youngest mergers in the Toomre Sequence.
\end{abstract}

Keywords. galaxies: formation, galaxies: interactions, galaxies: star clusters
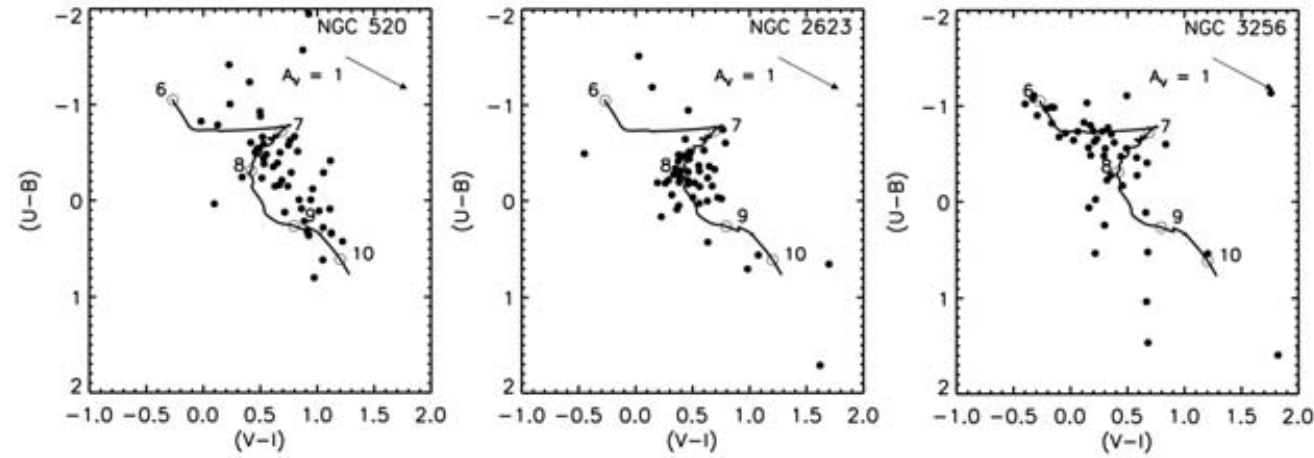

Figure 1: $U B V I$ color-color plots of the 50 brightest cluster candidates in each merger. Overlaid are the Bruzual-Charlot 2003 tracks for a single-burst populations with Salpeter IMF and Solar metallicity. The open circles mark the ages in log years.

- The ages of the brightest cluster candidates in NGC 520 show a large scatter, likely from dust, with most $\geqslant 100 \mathrm{Myr}$, and some $\geqslant 1$ Gyr. The older ages are confirmed by the absence of $\mathrm{H} \alpha$ emission. Clusters in the south-east tidal tail (not shown) are 100-250 Myr, providing a dynamical constraint on the age of the merger.

- The cluster candidates in NGC 2623 show a tight age range of 100-250 Myr.

- NGC 3256 has the youngest cluster population and fewest clusters > $100 \mathrm{Myr}$, despite being ranked as the most advanced of the three mergers along the Toomre Sequence.

- The slopes of the luminosity functions (not shown) for NGC 2623 and NGC 3256 are power-laws consistent with results from the Antennae. NGC 520 has a steeper slope, possibly influenced by large amounts of dust "masking" its cluster population. 